\title{
Intervencionismo endoscópico en la pancreatitis crónica
}

\section{Endoscopic Intervention in Chronic Pancreatitis}

\author{
Rodrigo Castaño Llano, ${ }^{1 *}$ Everson L. A. Artifon. ${ }^{2}$
}

\footnotetext{
1 Cirugía gastrointestinal y endoscopia. Jefe de Posgrado de Cirugía General, Universidad Pontificia Bolivariana. Profesor titular del grupo de gastrohepatología, Universidad de Antioquia. Cirugía y endoscopia del Instituto de Cancerología, Clínica las Américas. Medellín, Colombia.

2 Profesor del Departamento de Cirugía de la Facultad de Medicina de la Universidad de Sao Paulo (FMUSP). Coordinador de la unidad de endoscopia biliopancreática, FMUSP. Vicepresidente de la Sociedad Interamericana de Endoscopia Digestiva (SIED; 2017-2018) Sao Paulo, Brasil.
}

*Correspondencia: rcastanoll@hotmail.com.

Fecha recibido: $\quad 02 / 04 / 18$

Fecha aceptado: 30/07/18

\begin{abstract}
Resumen
La pancreatitis crónica es un trastorno irreversible y progresivo del páncreas caracterizado por inflamación, fibrosis y cicatrización. Las funciones exocrinas y endocrinas se pierden, lo que a menudo conduce al dolor crónico. La etiología es multifactorial, aunque el alcoholismo es el factor de riesgo más importante en los adultos. Si se sospecha pancreatitis crónica, la tomografía computarizada con contraste es la mejor modalidad de diagnóstico por imágenes. Aunque los narcóticos y los antidepresivos proporcionan el mayor alivio del dolor, más de la mitad de los pacientes eventualmente requiere una intervención por endoscopia o cirugía. La colangiopancreatografía retrógrada endoscópica es una alternativa eficaz para una variedad de terapias en el tratamiento de enfermedades benignas y malignas del páncreas. En los últimos 50 años, la endoterapia ha evolucionado hasta convertirse en la terapia de primera línea en la mayoría de las enfermedades inflamatorias agudas y crónicas del páncreas. A medida que avanza este campo, es importante que los gastroenterólogos mantengan un conocimiento adecuado de la indicación del procedimiento, mantengan el volumen de procedimiento suficiente para manejar la endoterapia pancreática compleja y comprendan enfoques alternativos a las enfermedades pancreáticas, incluidos el tratamiento médico, la terapia guiada por ecografía endoscópica, el manejo de las estenosis sintomáticas y cálculos, las intervenciones sobre el plexo celíaco y el drenaje de los pseudoquistes pancreáticos.
\end{abstract}

\section{Palabras clave}

Pancreatitis crónica, colangiografía endoscópica, cálculos pancreáticos, estenosis pancreática, pseudoquiste pancreático, bloqueo de plexo celíaco.

\begin{abstract}
Chronic pancreatitis is an irreversible and progressive disorder of the pancreas characterized by inflammation, fibrosis and scarring. Exocrine and endocrine functions are lost often leading to chronic pain. Its etiology is multifactorial, although alcoholism is the most important risk factor in adults. If chronic pancreatitis is suspected, computed tomography with contrast is the best imaging modality. Although narcotics and antidepressants provide the greatest pain relief, more than half of all patients eventually require intervention by endoscopy or surgery.

Endoscopic retrograde cholangiopancreatography (ERCP) is an effective alternative for a variety of therapies for treating benign and malignant diseases of the pancreas. In the last 50 years, endoscopic treatment has evolved to become the first-line therapy for most acute and chronic inflammatory diseases of the pancreas. As this field progresses, it has become important for gastroenterologists to keep their knowledge of indications for this procedure up-to-date and to perform a sufficient volume of procedures to allow them to manage complex pancreatic endoscopic therapy. Keeping up-to-date should include an understanding of alternative approaches to pancreatic diseases including medical treatment, therapy guided by endoscopic ultrasound, management of symptomatic stenoses and stones, interventions on the celiac plexus, and drainage of pancreatic pseudocysts.
\end{abstract}

\section{Keywords}

Chronic pancreatitis, endoscopic cholangiography, pancreatic stones, pancreatic stenosis, pancreatic pseudocyst, celiac pleural block. 


\section{INTRODUCCIÓN}

La pancreatitis crónica (PC) es una enfermedad diagnosticada cada vez más frecuentemente en la práctica médica. Se estima que la incidencia de la PC varía entre 5 y 14,4 casos por 100000 habitantes $(1,2)$. El dolor abdominal intratable y las anomalías morfológicas asociadas en el sistema pancreatobiliar son los principales factores determinantes del tratamiento endoscópico intervencionista en la PC (3). Los mecanismos de dolor en PC son múltiples y la evidencia experimental reciente sugiere que la hipertensión ductal pancreática puede activar células estrelladas pancreáticas, que a su vez pueden generar estrés oxidativo e inflamación subsecuente (4). Uno de los orígenes del dolor surge de la sumatoria de inflamación crónica y estrés oxidativo induciendo nocicepción, alodinia mecánica e hiperalgesia inflamatoria, neuropatía pancreática y neuroplasticidad periférica y central (5). En algunos pacientes, existen anormalidades estructurales dentro del ducto o del parénquima pancreático que pueden ser responsables o que pueden perpetuar este proceso. Dichas anormalidades son a menudo el objetivo para la intervención endoscópica o quirúrgica; sin embargo, su corrección no siempre se traduce en mejoría clínica, lo que subraya la naturaleza multimodal del síndrome de dolor de estos pacientes. La descompresión se puede realizar utilizando abordajes endoscópicos y quirúrgicos, con un enfoque endoscópico recomendado actualmente como la modalidad de primera línea por la Sociedad Europea de Endoscopia Gastrointestinal (ESGE) (6).

Es obligatorio realizar una evaluación morfológica meticulosa del Wirsung o conducto principal pancreático (CPP) para valorar la magnitud de la enfermedad y las alteraciones anatómicas locales que sugieran neoplasia antes de indicar la terapia endoscópica. Se debe descartar cáncer de páncreas, especialmente en pacientes mayores de 50 años, de sexo femenino, de raza blanca, con ictericia e insuficiencia exocrina manifiesta, y con ausencia de calcificaciones pancreáticas (7). El tamaño y distribución de los cálculos ductales pancreáticos pueden evaluarse mejor mediante tomografía axial computarizada (TAC), aunque la ecografía transabdominal también proporciona una evaluación bastante buena. La presencia de estenosis ductal pancreática, estenosis biliares y variantes anatómicas como el páncreas divisum se identifican mejor con la colangiopancreatografía por resonancia magnética (CPRM). La CPRM también es ventajosa con respecto al ultrasonido endoscópico (USE) y la TAC para diferenciar un pseudoquiste pancreático (PP) de una necrosis (walled-off necrosis) peri- o intrapancreática, en pacientes con PC que cursan con exacerbación aguda reciente (8).

La PC es responsable por un extenso uso de los cuidados de salud y está asociada con un costo socioeconómico desproporcionadamente alto (9). El manejo clínico de esta enfermedad es a menudo retador porque frecuentemente involucra la prescripción de opiáceos para el control del dolor, los cuales son altamente regulados y se asocian con numerosos efectos colaterales y problemas de dependencia. Los abordajes actuales de la terapia endoscópica para PC están dirigidos para el alivio de cálculos que obstruyan el ducto pancreático, estenosis del ducto pancreático, drenaje de PP, bloqueo del plexo celíaco (BPC) y estenosis biliares benignas. Cada una de estas técnicas y su contribución en el tratamiento de PC serán revisadas.

\section{INDICACIONES Y CONTRAINDICACIONES DE LA TERAPIA ENDOSCÓPICA EN PC}

El dolor intratable es la indicación más común para la terapia endoscópica pancreática en pacientes con PC y la modalidad de elección depende de la morfología:

- Los cálculos pequeños se pueden extraer mediante colangiopancreatografía retrógrada endoscópica (CPRE) con esfinterotomía pancreática, mediante el uso de balones y canastilla de Dormia. Los cálculos de más de $5 \mathrm{~mm}$ pueden fragmentarse mejor mediante litotricia extracorpórea con ondas de choque (LEOC) con o sin stent ductal pancreático (10).

- Actualmente, la LEOC ha sido recomendada como la primera opción por la ESGE (6).

- Las contraindicaciones para la LEOC son los múltiples cálculos a lo largo del Wirsung, cálculos aislados en la región de la cola, múltiples restricciones de Wirsung, presencia de ascitis de moderada a masiva, pseudoquiste de páncreas y masa de la cabeza pancreática (11).

- Las estenosis ductales pancreáticas pueden tratarse mediante esfinterotomía pancreática y stent con o sin dilatación.

- Los PP deben tratarse en presencia de infección, hemorragia intraquística sintomática, obstrucción biliar, obstrucción de la salida gástrica, saciedad precoz, dolor abdominal, pérdida de peso y aumento del tamaño del pseudoquiste. Se ha sugerido que el tratamiento profiláctico del pseudoquiste asintomático se puede considerar en presencia de compresión de los vasos principales, fístula pancreaticopleural, tamaño de pseudoquiste $>5 \mathrm{~cm}$ que no disminuye después de 6 semanas y espesor de la pared del pseudoquiste $>5 \mathrm{~mm}$ (12).

- El USE encuentra un lugar en el drenaje del CPP dilatado utilizando técnicas rendezvous cuando el acceso transpapilar no es posible (13).

- Tratamiento del dolor refractario a la intervención estándar; puede intentarse con BPC guiado por USE (14). 


\section{TERAPIA ENDOSCÓPICA PARA LOS CÁLCULOS DUCTALES PANCREÁTICOS}

Los cálculos del ducto pancreático son concreciones biominerales que pueden obstruir los ductos pancreáticos. Esto puede resultar en incremento de la presión del ducto pancreático activando un proceso inflamatorio que provoca fibrosis. El objetivo de la terapia endoscópica en pacientes con enfermedad calculosa es aliviar la obstrucción con la remoción de los cálculos (15) (Figura 1).

Con los años, las técnicas para fragmentar y eliminar los cálculos ductales pancreáticos han evolucionado (16). Estas técnicas pueden requerir un acceso intraductal como la litotricia mecánica, litotricia electrohidráulica y litotricia guiada por láser; o una intervención extracorpórea como la LEOC.

La litotricia mecánica intraductal, que se realiza mediante un litotriptor mecánico por vía endoscópica, es técnicamente desafiante con una alta tasa de complicaciones y actualmente rara vez se usa. La litotricia electrohidráulica se realiza bajo visualización pancreatoscópica directa utilizando un sistema endoscópico madre-hijo y tiene la ventaja de suministrar energía a un área focalizada de los cálculos (17). Aunque haya modificaciones técnicas que evolucionan con el tiempo, como el uso de un solo operador del SpyGlass y litotricia con láser de holmio, los estudios en la literatura son limitados (18). Además, la disponibilidad por costos y experticia de los operadores de estas técnicas impide la utilización generalizada.

La LEOC es actualmente la modalidad de primera línea para tratar cálculos ductales pancreáticos obstructivos grandes $(>5 \mathrm{~mm})$ dolorosos, particularmente para aquellos localizados en las regiones de cabeza y cuerpo (6). El objetivo es reducir los cálculos a fragmentos menores de $3 \mathrm{~mm}$. Las piedras fragmentadas generalmente se eliminan con una CPRE y esfinterotomía pancreática después de la LEOC. Un stent ductal pancreático concomitante se puede utilizar en presencia de una estenosis local.
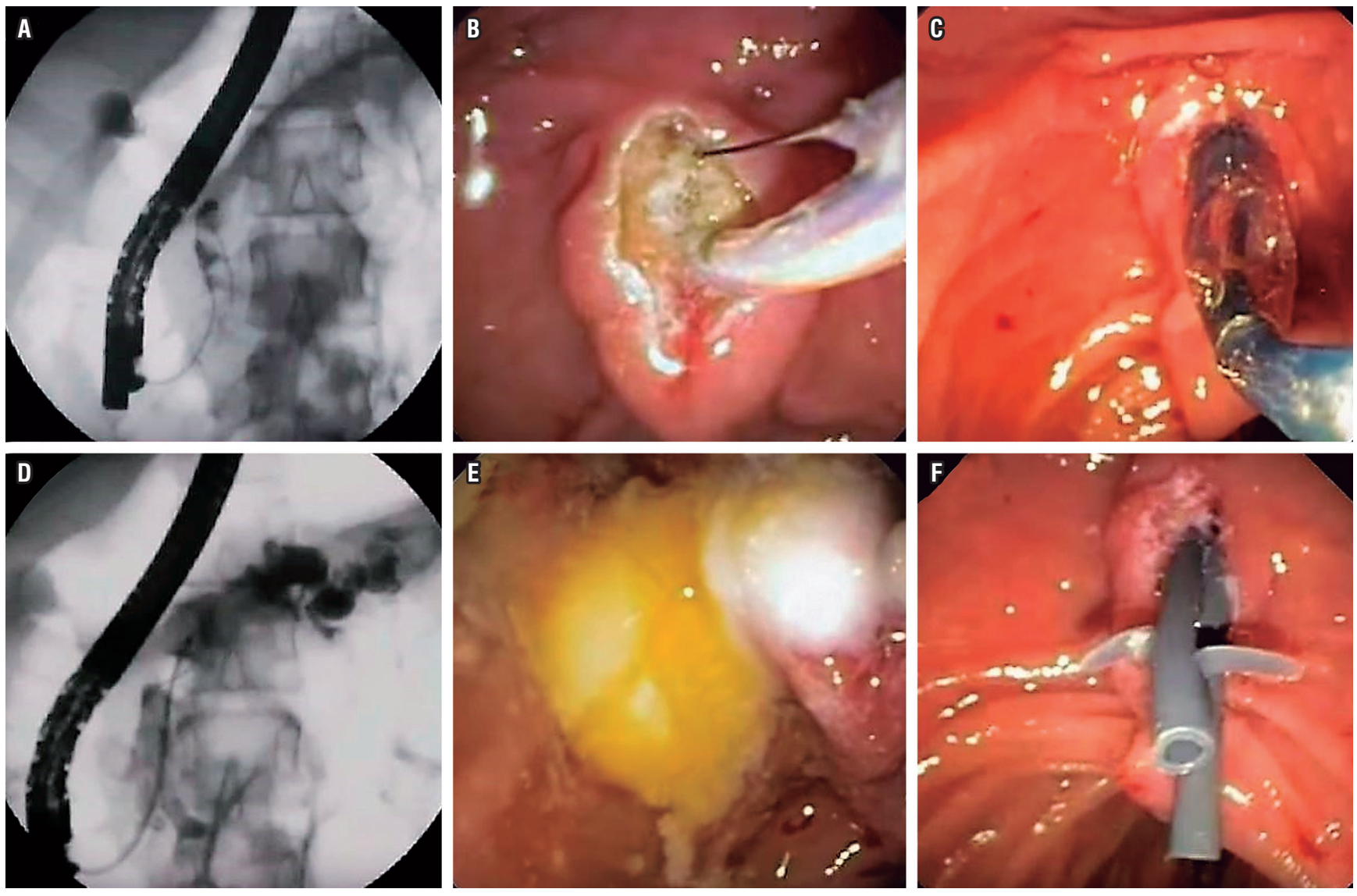

Figura 1. Manejo de cálculos en el CPP en paciente con PC. A. La pancreatografía muestra varios cálculos en la cabeza. B. Esfinterotomía del pancreático. C. Dilatación con balón de la esfinterotomía. D. Paso de balón de extracción. E. Cálculos pancreáticos extraídos. F. Paso de 2 stents pancreáticos de 7 Fr por $10 \mathrm{~cm}$. 
Para los cálculos radiotransparentes, una esfinterotomía pancreática previa a la LEOC con colocación de un tubo de drenaje nasopancreático puede ayudar a orientar con precisión la LEOC (10). En presencia de cálculos ductales múltiples, el cálculo que se encuentra más cerca del orificio del CPP debe ser el objetivo principal. Los cálculos intraductales aislados localizadas en la cola no necesitan tratamiento con LEOC, porque es poco probable que estas concreciones den como resultado una presión ductal ascendente suficiente como para causar dolor. Además, los intentos de fragmentar los cálculos ductales en la cola podrían provocar una lesión esplénica colateral.

La cirugía derivativa es una alternativa para el manejo del dolor y lograr la descompresión de los ductos, y se compara favorablemente con la endoterapia sin LEOC. Sin embargo, debido a la morbilidad asociada con la cirugía, como la cirugía de Frey (Figura 2), esta se reserva generalmente para pacientes en quienes han fallado los tratamientos no quirúrgicos (19).

\section{TERAPIA ENDOSCÓPICA PARA LAS ESTENOSIS DEL DUCTO PRINCIPAL}

Se observan estenosis en el CPP principal hasta en el $18 \%$ de los pacientes con PC y la prioridad es descartar malignidad. Similar al principio y a los problemas creados por cálculos obstructivos, se piensa que esta estenosis contribuye a los síntomas porque causa hipertensión ductal pancreática; entonces, la terapia es dirigida a mejorar estos segmentos estrechos para descomprimir al sistema ductal. El abordaje usualmente involucra la esfinterotomía pancreática, seguida por la dilatación de la estenosis y la colocación de un stent en el ducto pancreático (Figura 3). El alivio inmediato y sostenido del dolor con este tratamiento
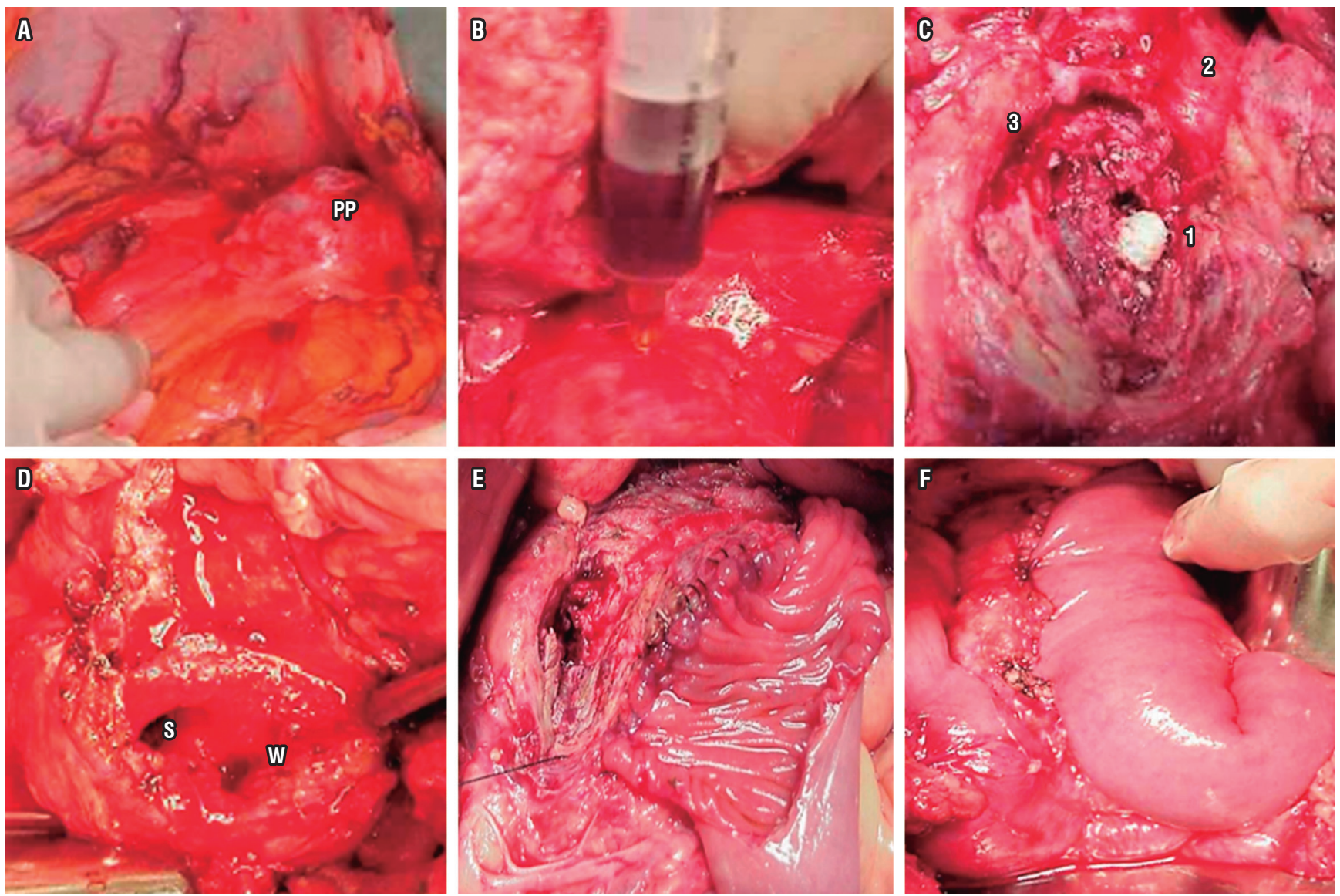

Figura 2. Cirugía de Frey en la PC. A. Abordaje del PP en la transcavidad. B. Punción de la cara anterior del cuerpo pancreático para ubicar el conducto pancreático principal. C. Cálculos (1) en el Wirsung abierto (2), se aprecia otro cálculo en el Santorini (3). D. Wirsung abierto (W) y conducto de Santorini (S) en la cabeza pancreática. E. Anastomosis parcial del asa yeyunal aislada a la cara anterior del páncreas. F. Anastomosis terminada en 2 planos. 

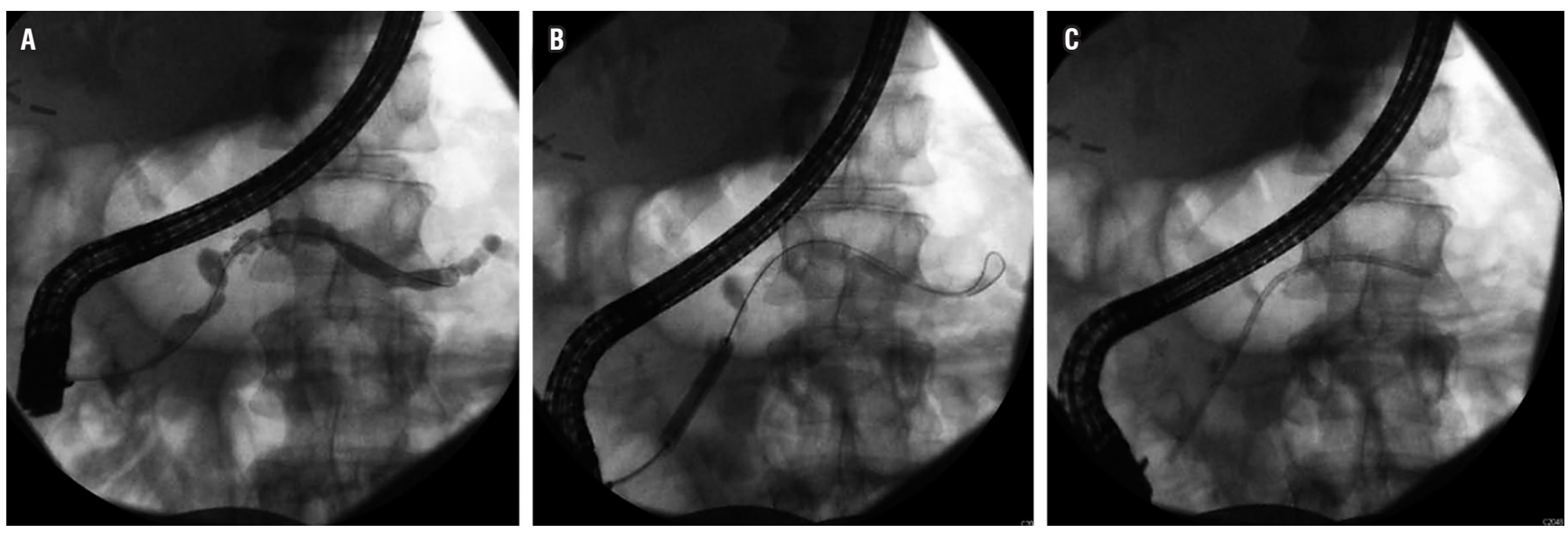

Figura 3. Manejo de estenosis benigna con dilatación y stent en un paciente con PC. A. Estenosis en la cabeza del páncreas. B. Dilatación con balón de la estenosis. C. Paso del stent pancreático.

se ha reportado en $65 \%-95 \%$ y $32 \%-68 \%$ de los pacientes, respectivamente (20).

Actualmente, la colocación de un solo stent de polietileno de $10 \mathrm{Fr}$ con cambios sucesivos por 1 año, incluso en ausencia de síntomas, es el tratamiento de primera línea de la estenosis dominante del CPP (6).

A pesar de que los stents de plástico rectos o con cola de cerdo se han usado ampliamente para tratar las estenosis de los ductos pancreáticos, aún no existe un stent ideal. Hasta el momento, se han realizado varias modificaciones en la tecnología del stent pancreático, del tipo S, con flaps laterales, de diámetro o celdas irregulares (bumpy stent), o incluso biodegradables (21). Sin embargo, los diferentes stents se han probado en modelos animales o en ensayos clínicos a corto plazo con tamaños de muestra pequeños. Los datos sobre la validación de estos resultados y los datos a largo plazo sobre la eficacia y la seguridad deben esperarse antes de que estos stents se puedan utilizar de forma rutinaria.

Una técnica en estudio para tratar las estenosis del ducto principal que persisten más allá de los 12 meses después del manejo con un único stent plástico es el despliegue de múltiples stents de plástico colocados lado a lado simultáneamente (22).

En un estudio colaborativo multicéntrico internacional se demostró que el drenaje del ducto pancreático por USE en las estenosis pancreáticas después de una CPRE fallida es un procedimiento seguro, mínimamente invasivo con respecto a la cirugía e incluso más efectivo (23).

\section{TERAPIA ENDOSCÓPICA PARA PP}

Los PP son colecciones de jugo pancreático encapsulado, con una pared inflamatoria bien definida, que se desarro- llan en el $20 \%-40 \%$ de los pacientes con PC. A diferencia de los PP de origen agudo, los de la PC raramente remiten espontáneamente. La intervención está indicada cuando los síntomas abdominales persisten (p. ej. dolor abdominal, náuseas, vómitos, saciedad precoz y pérdida de peso), obstrucción de la salida gástrica o biliar, o infección. Algunos tratamientos se han propuesto para colecciones asintomáticas persistentes que son grandes o que tienen una pared gruesa (reconociendo que ser asintomático no es común para quistes $>6 \mathrm{~cm}$ ) y aquellos que pueden estar asociados con fístula, cambios del ducto pancreático principal o con cálculos del ducto pancreático. El drenaje endoscópico de los pseudoquistes se puede realizar mediante abordajes transmurales y transpapilares (24).

Es importante evaluar la anatomía ductal anormal, incluidas las fugas ductales y si existe o no comunicación con el pseudoquiste, con una CPRM o CPRE previa al tratamiento. Un aumento en el tamaño del pseudoquiste en las imágenes sugiere comunicación ductal con el pseudoquiste. La presencia de obstrucción ductal debe manejarse antes de la terapia endoscópica para que la terapia de los pseudoquistes logre una mayor tasa de éxito y de este modo evitar la recurrencia. De forma similar, los pseudoaneurismas arteriales, si se detectan, deben embolizarse antes de realizar la terapia endoscópica porque la mortalidad asociada con hemorragia por pseudoaneurismas cercanos a los pseudoquistes es alta (25).

El drenaje transpapilar durante la CPRE es más útil para pseudoquistes pequeños, solitarios y comunicantes situados en relación con la cabeza y el cuerpo del páncreas. Este tipo de drenaje también se ha mostrado factible y útil en PP grandes y múltiples, sin mostrar unos resultados mejores o inferiores al drenaje transmural (26). 
El drenaje transmural se puede realizar creando una comunicación entre el pseudoquiste y el estómago (cistogastrostomía) o el duodeno (cistoduodenostomía). Después de la punción, se deben colocar al menos 2 stents de plástico con doble cola de cerdo a través de la punción para mantener abierta la abertura entre el pseudoquiste y el estómago/duodeno. El stent no debe retirarse antes de 2 meses de la inserción y una imagen de corte transversal debe realizarse obligatoriamente para evaluar la resolución del quiste antes de la remoción del stent (27).

Si hay evidencia de imágenes de un CPP seccionado, el stent debe mantenerse in situ indefinidamente para lograr los mejores resultados. Un intento por pasar con el stent la ruptura del conducto, si es posible, también se asocia con un buen éxito a largo plazo (28).

Antes del uso de USE en el drenaje del pseudoquiste, la presencia de una compresión en la pared gástrica o duodenal era esencial, y a través de ella se realizaba la punción/ drenaje. Bajo guía ecoendoscópica lineal, el drenaje se puede realizar incluso para pseudoquistes no abultados que se encuentran incluso más allá de la luz gástrica o duodenal con resultados superiores (29). El USE también puede ayudar a delimitar un área avascular para la punción, lo que es de ayuda adicional en pacientes con colaterales vasculares extensas secundarias a hipertensión portal. El USE ofrece una ayuda adicional para distinguir los pseudoquistes de las neoplasias quísticas (30).

\section{TERAPIA ENDOSCÓPICA EN LA ESTENOSIS BILIAR POR PC}

La estenosis biliar benigna se encuentra en muchos pacientes con PC. Su rango de prevalencia estimada es amplio, probablemente basado en el contexto clínico en el cual se ha estudiado, pero puede estar presente en el $3 \%-46 \%$ (31). En general, se presentan como una estrechez circunferencial fibrocalcificada que usualmente se desarrolla dentro de la porción pancreática del ducto biliar común, esto hace posible la obstrucción biliar que se desarrolla de la compresión extrínseca relacionada con el edema pancreático o la colección de fluido. Cuando las estenosis son identificadas es importante excluir malignidad. Los pacientes pueden experimentar dolor, náuseas, pérdida de peso, ictericia, prurito y el $10 \%$ puede progresar y desarrollar colangitis o cirrosis biliar (32).

Una indicación clara de intervención endoscópica en PC es la colangitis; sin embargo, su indicación como un procedimiento preventivo es menos clara. Existen numerosas observaciones de pacientes con colestasis por estenosis biliar benigna relacionada con PC manejadas exitosamente sin drenaje biliar, aunque no está claro cómo predecir quié- nes van a progresar o a desarrollar otras complicaciones y si es posible que la fibrosis hepática regrese después del drenaje biliar exitoso. La principal indicación para la intervención endoscópica se ha adoptado de la conducta quirúrgica y, además de la presencia de síntomas, se incluyen la cirrosis biliar secundaria, cálculos en el ducto biliar común, progresión de la estenosis biliar basada en el incremento de la dilatación proximal ductal biliar, ictericia persistente (más de 1 mes) o fosfatasa alcalina mayor de 2 a 3 veces el límite superior normal (6).

\section{USE DESPUÉS DE UNA CPRE FALLIDA}

El desarrollo del USE de matriz lineal ha permitido nuevos enfoques para el drenaje del sistema pancreatobiliar cuando falla la CPRE convencional.

Después de la descripción inicial de Harada (33), varios estudios evaluaron la viabilidad y la eficacia del drenaje pancreatobiliar dirigido por USE. El principio fundamental es perforar el CPP guiado por el USE a través de la pared gástrica o duodenal con una aguja de calibre grande. Una vez que se logra el acceso exitoso al Wirsung, el drenaje ductal se puede realizar mediante técnicas de rendezvous o la ruta transmural (34). En vista del desafío técnico planteado por los procedimientos de rendezvous guiados por USE y la alta frecuencia de las complicaciones, actualmente se recomienda solo para pacientes seleccionados en centros de atención terciaria con la infraestructura y experiencia adecuadas (35).

\section{BPC GUIADO POR ECOGRAFÍA ENDOSCÓPICA}

El BPC es una opción de tratamiento dirigido a la disrupción de la vía aferente de transmisión del dolor desde el páncreas (36). Típicamente, implica inyectar el plexo celíaco con una mezcla de anestésico local y un corticosteroide (esto es distinto de la neurólisis del plexo celíaco con inyección de etanol, lo cual no debe usarse en el tratamiento de la enfermedad pancreática benigna) (37). Los esteroides son utilizados como un sustituto del etanol para prolongar el efecto del tratamiento. La combinación de bupivacaína y triamcinolona fue también el régimen seleccionado en el mayor estudio prospectivo sobre BPC guiado por USE (38).

El BPC puede realizarse por vía percutánea; sin embargo, el procedimiento guiado por USE tiene mejores resultados y menor riesgo de complicaciones, como la paraplejia, que se asocia con la técnica percutánea (14). En vista de la dudosa eficacia (alivio del dolor de corta duración, si es que hay alguno) y complicaciones frecuentes, el BPC debe mantenerse como terapia de rescate o puente para los pacientes que no responden a la terapia médica y endoscó- 
pica convencional y no son candidatos quirúrgicos ideales (39). Aunque la neurólisis ganglionar celíaca guiada por USE con alcohol absoluto está justificada en el cáncer de páncreas, debe evitarse en PC, ya que la fibrosis resultante de la inyección de alcohol podría dificultar técnicamente la cirugía posterior.

Ensayos aleatorios comparativos han demostrado que el BPC guiado por USE es superior a el BPC guiado con el fluoroscopio o al BPC guiado por TAC en términos tanto del alivio del dolor como de la preferencia del paciente. El número de pacientes que puede esperarse que experimente mejoría del dolor es alrededor de $50 \%-60 \%$ con BPC guiada por USE, pero un problema mayor es que la mejoría del dolor no es usualmente persistente y muchos pacientes pierden el beneficio luego de varios meses. Algunos pacientes pueden responder al BPC secuencial, pero esta estrategia de manejo no está probada y acumula un riesgo con cada intervención (12).

\section{COMPLICACIONES}

A pesar de que los procedimientos de litotricia intraductal mecánica y electrohidráulica están asociados con un mayor riesgo de complicaciones, la LEOC es un procedimiento relativamente seguro. Las complicaciones habituales de LEOC incluyen pancreatitis aguda (PA), lesión esplénica, petequias cutáneas, hemorragia, calle de piedras y la perforación, siendo la PA la más importante y frecuente. En un estudio reciente que involucró 1470 procedimientos, por una parte, la LEOC informó una tasa de complicaciones general de $6,7 \%$. El estudio documentó una odds ratio (OR) de 1,28 para el desarrollo de complicaciones postLEOC en presencia de páncreas divisum y el intervalo entre el diagnóstico de PC, respectivamente. Por otra parte, el género masculino surgió como un posible factor de protección independiente contra las complicaciones moderadas a graves, con un OR de 0,19 (40).

Los problemas comunes encontrados con los stents pancreáticos incluyen la migración y la obstrucción. La duración de la permeabilidad de los stents pancreáticos suele oscilar entre 6 y 12 meses (41).

Las complicaciones del drenaje endoscópico del pseudoquiste incluyen hemorragia, infección y fuga retroperitoneal, respectivamente, observadas en aproximadamente $4 \%$ de los pacientes, aunque la mortalidad suele ser baja (0,5 \%) (42). Las complicaciones, como la hemorragia y la ruptura, son menores en el método transpapilar, pero el riesgo de infección es mayor.

Las complicaciones del BPC comúnmente incluyen diarrea transitoria, exacerbación del dolor, hipotensión, ocasionalmente infecciones, y raramente la muerte (43).

\section{RESULTADOS}

\section{Litotricia extracorpórea con ondas de choque (LEOC)}

La eficacia de la LEOC suele medirse en términos de fragmentación completa de cálculos, eliminación de cálculos y

Tabla 1. Estudios del siglo XXI que muestran los resultados de la LEOC con o sin colangiografía endoscópica retrógrada para aliviar el dolor y aclarar cálculos del conducto pancreático principal en la PC

\begin{tabular}{lccccc}
\hline \multicolumn{1}{c}{ Autorlaño } & $\mathbf{n}$ & Meses & Tratamiento & $\begin{array}{c}\text { Alivio inicial-\% (alivio } \\
\text { total-\%) }\end{array}$ & Aclaramiento (\%) \\
\hline Brand, 2000 (45) & 48 & 7 & LEOC + CPRE & 82 & 44 \\
Kozarek, 2002 (46) & 40 & 30 & LEOC + CPRE & 80 & SD \\
Fernbacher, 2004 (47) & 125 & 29 & LEOC + CPRE & $(48)$ & 64 \\
Delahaye, 2004 (48) & 56 & 173 & LEOC + CPRE & $85(45)$ & 48 \\
Iniu, 2005 (49) & 237 & 44 & LEOC + CPRE & 91 & 73 \\
& 318 & & LEOC & & 70 \\
Dumonceau, 2007 (50) & 29 & 52 & LEOC + CPRE & 52 & SD \\
Seven, 2012 (51) & 26 & & LEOC & & SD \\
Tandan, 2013 (52) & 120 & 51 & LEOC + CPRE & 51 & 77 \\
Vaysse, 2016 (53) & 636 & 96 & LEOC + CPRE & 96 & SD \\
& 91 & 6 & LEOC + CPRE & 76 & \\
Korpela, 2016 (10) & 41 & & LEOC & & SD \\
\hline
\end{tabular}

SD: sin dato. 
alivio del dolor. Recientemente, se ha demostrado que los cálculos solitarios y de baja densidad son predictores independientes del aclaramiento completo por LEOC (44). La Tabla 1 enumera los estudios que informaron la eliminación completa de los cálculos del conducto y alivio del dolor en respuesta a LEOC con o sin CPRE.

Tandan ha demostrado recientemente un alivio completo del dolor en $68,7 \%$ y $60,3 \%$ de los pacientes en seguimiento intermedio ( $2-5$ años) y a largo plazo ( $>5$ años), respectivamente, después de la LEOC en una cohorte de 636 pacientes (52). Se observó el aclaramiento completo de los conductos en 77,5\% y $76 \%$ de los pacientes en los grupos de seguimiento intermedio y a largo plazo, respectivamente. Aunque el $14,1 \%$ de pacientes en el grupo de seguimiento intermedio y el $22,8 \%$ en el grupo a largo plazo tuvieron recurrencia de cálculos, solo el 3,8 \% de los pacientes en el seguimiento intermedio, mientras que ninguno en el seguimiento a largo plazo, requirió repetición de LEOC. Este estudio sugirió que, si la LEOC se inicia desde el principio, es probable que el alivio del dolor persista durante un tiempo más prolongado.

Seven y colaboradores (51), en un estudio con 120 pacientes, mostraron alivio del dolor en $85 \%$ de los pacientes después de un seguimiento medio de 4,3 años. Se observó alivio completo del dolor en el $50 \%$ de los pacientes y hubo una mejoría significativa en los puntajes de calidad de vida (escala visual analógica $[E V A] 7,3[2,7]$ frente a 3,7 [2,4]; $\mathrm{p}<0,001)$. La proporción de pacientes sin dolor seguidos durante 4 años fue significativamente más alta que aquellos que se sometieron a cirugía (61\% frente a $21 \%$; $\mathrm{p}=0,009)$. El período de seguimiento más prolongado en este estudio fue por más de 7 años.

Se ha demostrado que el uso de secretina antes de LEOC da como resultado una mayor eliminación de cálculos en el estudio realizado por Choi y colaboradores (54). En este estudio, la administración intravenosa de $16 \mu \mathrm{g}$ de secretina antes de la LEOC resultó en $63 \%$ de aclaramiento de cálculos en comparación con $46 \%$ cuando la secretina no fue usada. La regresión logística múltiple sugirió que el uso de la secretina y la colocación de stent pancreático previo a la LEOC son predictores independientes de la depuración completa o casi total del CPP.

\section{Stent del conducto pancreático para estenosis}

La Tabla 2 recopila los estudios más importantes del siglo XXI que han evaluado el papel del stent ductal pancreático para las estenosis del CPP.

El éxito clínico a largo plazo después de la colocación de un stent en la estenosis del CPP se mide por la ausencia de dolor al año después de la extracción del stent. No es necesario un stent adicional si se demuestra la salida del contraste previo a la anastomosis en 1 a 2 minutos y el paso fácil de un catéter $6 \mathrm{Fr}$ a través de la estenosis (11).

El stent pancreático es técnicamente exitoso en $85 \%$ a $98 \%$ de los casos y se asocia con alivio inmediato del dolor en $65 \%$ a $95 \%$ de los pacientes, y se mantiene en $32 \%$ a $68 \%$ en el seguimiento de hasta 14 a 58 meses (66). En un

Tabla 2. Estudios del siglo XXI con manejo por colangiografía endoscópica de las estenosis pancreáticas con stents en pacientes con PC

\begin{tabular}{lccccc}
\hline \multicolumn{1}{c}{ Autor/año } & $\mathbf{n}$ & Meses & Tipo de stent & $\begin{array}{c}\text { Alivio de estenosis/ } \\
\text { dolor (\%) }\end{array}$ & $\begin{array}{c}\text { Necesidad de cirugía } \\
\text { (\%) }\end{array}$ \\
\hline Morgan, 2003 (55) & 25 & SD & Plástico & $70 / 65$ & SD \\
Vitale, 2004 (56) & 89 & 43 & Plástico & SD/68 & 12 \\
Eleftherladis, 2005 (57) & 100 & 69 & Plástico & SD/62 & 4 \\
Ishihara, 2006 (58) & 20 & 21 & Plástico & $40 / 90$ & SD \\
Weber, 2007 (59) & 17 & 24 & Plástico & SD/83 & SD \\
Park, 2008 (60) & 13 & SD & Metálico & $100 / S D$ & SD \\
Moon, 2010 (61) & 32 & SD & Metálico & $100 / S D$ & 0 \\
Seza, 2011 (62) & 20 & 36 & Plástico & SD/85 & 0 \\
Giacino, 2012 (63) & 10 & 19 & Metálico & $100 / 60$ & 0 \\
Weber, 2013 (64) & 17 & 60 & Plástico & SD/57 & 7 \\
Ogura, 2016 (65) & 13 & 8 & Metálico & $85 / 92$ & 0 \\
Cahen, 2017 (21) & 19 & 12 & Reabsorbible & $58 / 52$ & 10 \\
Serie actual & 11 & 48 & Plástico & $45 / 45$ & 36 \\
\hline
\end{tabular}

SD: sin dato. 
estudio reciente de 17 pacientes que se sometieron a endoprótesis pancreática, el $57 \%$ permaneció completamente libre de dolor (sin recaída) luego de 5 años (64).

Se ha demostrado que en las estenosis pancreáticas la colocación de stents plásticos múltiples tiene tasas de éxito técnico y funcional de $100 \%$ y 94,7 \%, respectivamente, junto con el alivio del dolor en $84,2 \%$. La migración y reintervención del stent se observaron en $10,5 \%$ y $15,8 \%$, respectivamente (67).

El uso de stents metálicos se asoció con el $100 \%$ de éxito técnico y funcional. Aunque el $85,2 \%$ de los pacientes no presentó dolor en el seguimiento a corto plazo, se observaron complicaciones en el $26,8 \%$, la migración del stent en el 8,2 \% y la reintervención en el 9,8\% de pacientes (66). Actualmente, se recomienda el uso de stents metálicos solo en ensayos clínicos con intercambios planificados dentro de 1 año porque la permeabilidad de estos stents en el CPP se limita a esa duración.

\section{Drenaje de pseudoquistes pancreáticos}

Aunque la morbilidad relacionada con el procedimiento y la recurrencia del pseudoquiste son similares con el drenaje endoscópico y quirúrgico a largo plazo, la mortalidad relacionada con el procedimiento es significativamente menor con el drenaje endoscópico $(0,2 \%$ frente a $2,5 \%)$. Otras variables que se han encontrado significativamente mejores con la terapia endoscópica en comparación con la cirugía incluyen el costo, la duración de la estadía en el hospital y la calidad de vida hasta 3 meses después del drenaje (68).

Se ha encontrado que tanto el drenaje transpapilar como el transmural de los PP tienen un éxito similar a largo plazo, mientras que el primero tiene una morbilidad menor $(1,8$ $\%$ frente a $15,4 \% ; p<0,008)$. Aunque el drenaje transmural del pseudoquiste puede realizarse mediante drenaje convencional y guiado por USE, la tasa de éxito es mayor con el drenaje guiado por USE porque no requiere la presencia de una compresión intraluminal. Los resultados son más favorables para el pseudoquiste localizado en la cabeza del páncreas (11) (Figura 4).

El fracaso terapéutico para el drenaje endoscópico del $\mathrm{PP}$ se encontró independientemente asociado con la colocación de un único stent (recto) y la duración del stent de menos de 6 semanas en un estudio retrospectivo. Un ensayo aleatorizado controlado reciente ha demostrado que la recurrencia del pseudoquiste se asoció con la remoción temprana del stent (69).

La mayoría de los estudios ha evaluado el papel de la terapia endoscópica en el drenaje de pseudoquiste resultante tanto de PA como de PC. Ahora se reconoce cada vez más, después de la revisión de la clasificación de Atlanta (70), que los PP son poco comunes en PA (71). Es probable que muchos de los llamados pseudoquistes en pacientes con PA en esos estudios fueran realmente una necrosis amurallada. Por lo tanto, los resultados obtenidos de los estudios con drenaje de pseudoquiste son actualmente mejores si solo se consideran los pacientes con PC con verdaderos PP.

\section{Estenosis biliar por PC}

El manejo endoscópico de la estenosis biliar benigna en la PC usualmente involucra la colocación de un stent biliar mediante la CPRE. La dilatación sola con balón se realiza poco porque estas estenosis no se resuelven fácilmente y la reestenosis con recaída clínica no es infrecuente después de la remoción del stent. La opción actual es la de colocar uno o varios stents plásticos o colocar un stent metálico autoexpandible (SMA). El promedio de éxito a largo plazo (usualmente definido como resolución de la estenosis) con la colocación de stent plástico biliar endoscópico puede estar alrededor del $37 \%$ a los 32 meses (72). En la misma revisión, el rango de permeabilidad del SMA fue de $37 \%$ a $100 \%$ sobre una media de seguimiento de 45 meses. En una revisión sistemática amplia que presentó una comparación entre estos 2 abordajes, el éxito clínico fue mayor con los SMA no cubiertos ( $80 \%)$ que con un solo stent plástico (36\%), pero los estudios seleccionados no presentan datos suficientes sobre el uso de stents múltiples (73). Sin embargo, otras experiencias han mostrado alta proporción de éxito con múltiples stents plásticos; la opinión de expertos favorece este abordaje inicial y no apoya el uso rutinario de SMA biliar para esta indicación (6). Unos de los mayores problemas con la colocación de stent biliar son la obstrucción y la infección (Figura 5).

La frecuencia con la que deben ser programados los pacientes para intercambio de stent con el fin de prevenir este problema es también una pregunta habitual (como es variable la adherencia del paciente a esta recomendación). A menudo, a los pacientes se les plantea retornar en intervalos de 3 a 4 meses, pero la baja proporción de oclusión con múltiples stents plásticos puede permitir la prolongación del intervalo de cambio (74). Tampoco existe un consenso sobre el momento en el que la terapia endoscópica debe ser realizada antes de considerar la cirugía, y este interrogante debería ser discutido multidisciplinariamente.

Finalmente, las estenosis biliares benignas pueden encontrarse en muchos pacientes con PC, pero un paso temprano importante es la exclusión de malignidad. Estas estenosis tienen potencial para causar numerosos síntomas y provocar ictericia, colangitis y cirrosis biliar secundaria. La decisión de aplicar terapia se basa usualmente en el desarrollo de los síntomas o con la meta de prevenir infección y cirrosis. 
Ambas opciones, endoscópica y quirúrgica, están disponibles, pero se necesitan comparaciones directas. Las estenosis en su mayoría son recurrentes y pueden requerir varios tratamientos endoscópicos con balón dilatador y colocación de stents durante 1-2 años antes de alcanzar un desenlace más duradero. La selección óptima del stent continúa siendo evaluada, pero los resultados con el uso de stents múltiples plásticos es equivalente a los SMA. El
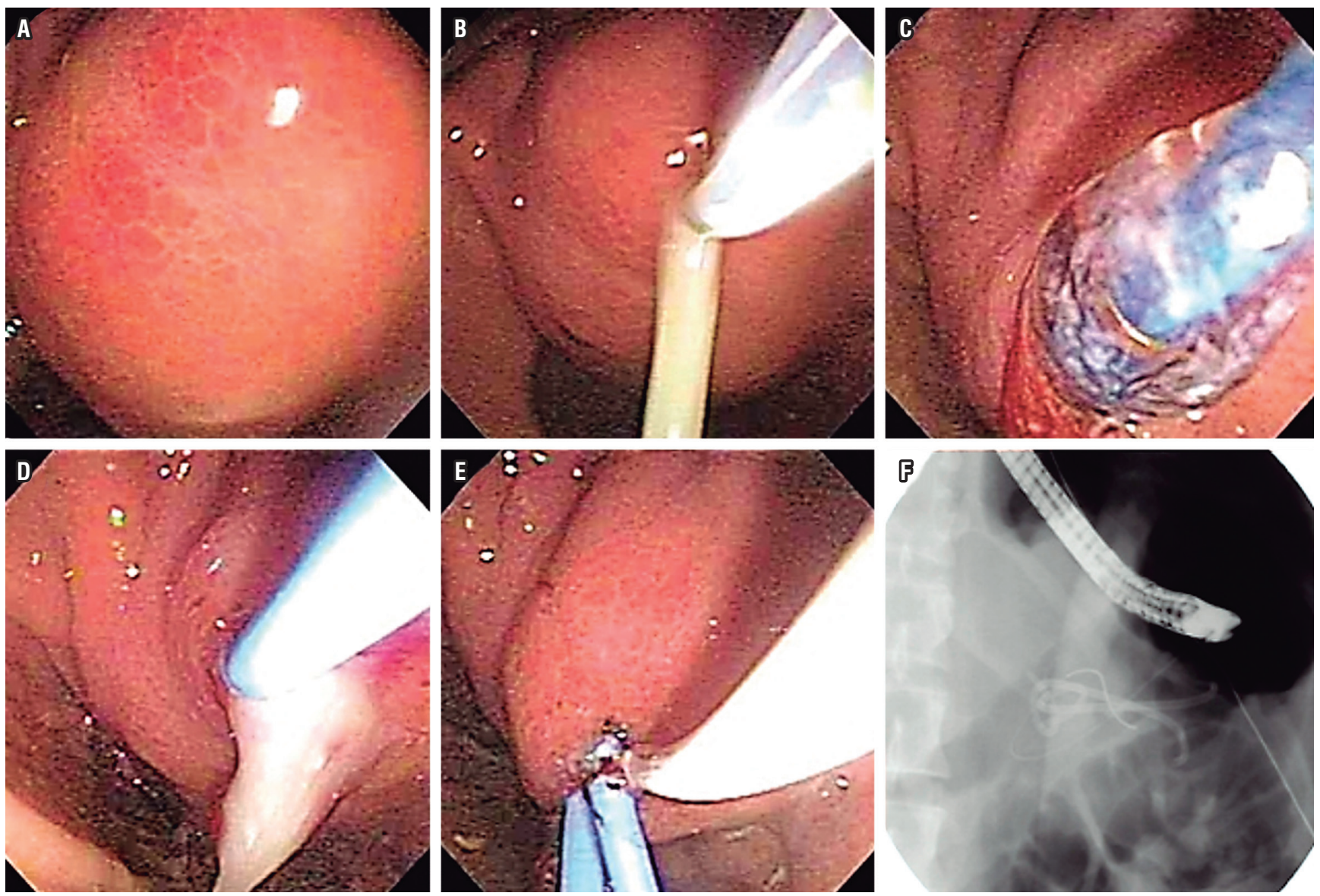

Figura 4. Drenaje endoscópico de PP en PC. A. PP que abomba en el antro gástrico. B. Punción con aguja de precorte con salida de pus. C. Dilatación transmural con balón hasta $10 \mathrm{~mm}$. D. Paso de primer catéter cola de cerdo 7 Fr. E. Paso del segundo catéter cola de cerdo. F. Aspecto fluoroscópico del doble drenaje.
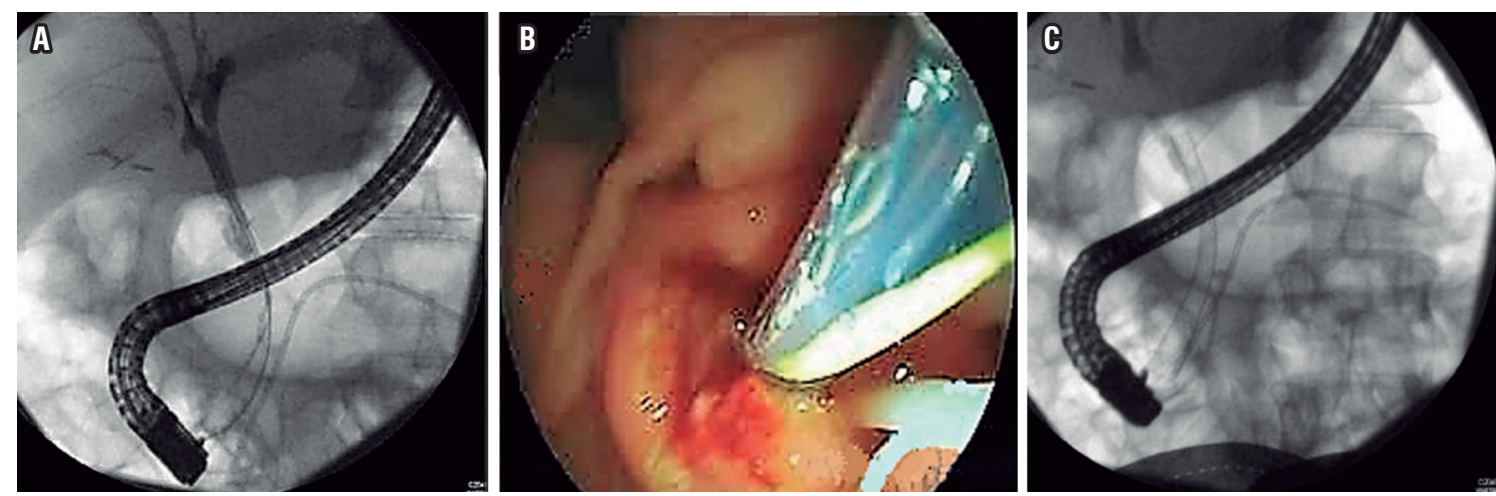

Figura 5. Manejo endoscópico de la estenosis biliar por PC. A. Colangiograma con estenosis biliar distal. B. Paso endoscópico de stent biliar metálico. C. Stent biliar metálico expandido y stent plástico en el páncreas. 
intercambio de los stents también debe realizarse cada pocos meses y la pobre adherencia a estos cambios de stents aumenta los riesgos de eventos adversos (75).

\section{Acceso guiado por USE y drenaje después de una CPRE fallida}

Un estudio reciente de Shah y colaboradores (76) reportó una tasa de éxito hasta del $75 \%$ para el procedimiento pancreático de rendezvous guiado por USE. Otro estudio multicéntrico español de 125 pacientes mostró un éxito técnico y clínico global de $67,2 \%$ y $63,2 \%$, respectivamente, para el acceso biliopancreático guiado por USE (77). En otro estudio de Ergun y colaboradores (78) se obtuvieron reducciones significativas en la puntuación del dolor y el diámetro del CPP, se observaron en el seguimiento a largo plazo (mediana: 38 meses [rango: 3-120]) de los pacientes que se sometieron con éxito a un drenaje guiado por USE.

\section{BPC guiado por ecografía endoscópica}

El papel del BPC, sea percutáneo o guiado por USE, para el alivio del dolor en PC ha sido controvertido (14). Incluso si es benéfico, el efecto es de corta duración, con $55 \%$ de pacientes que muestra mejoría después de 4 a 8 semanas y se reduce a $26 \%$ y $10 \%$ después de 12 y 24 semanas, respectivamente (38). Los resultados son aún más pobres para los pacientes menores de 45 años que se han sometido a cirugía pancreática previa (37).

\section{CONCLUSIONES}

La PC es una enfermedad desafiante cuyo síntoma primario es el dolor. Las razones más comúnmente descritas para la intervención endoscópica son los cálculos que obstruyen el ducto pancreático, estenosis del ducto pancreático, PP, bloqueo del plexo nervioso celíaco y el tratamiento de estenosis biliar benigna. La terapia endoscópica tiene un rol en cada uno de estos desórdenes y su capacidad se ha expandido o se ha refinado con el desarrollo de nuevas tecnologías como el USE, que permite abordajes extraanatómicos. En resumen:

- El síntoma primario de PC es el dolor.

- Los cálculos del ducto pancreático deben removerse si es posible endoscópicamente; no obstante, el dolor no siempre responde a la remoción de los cálculos.

- Las estenosis del ducto pancreático principal pueden ser manejadas con stent, pero siempre la malignidad debe ser excluida.

- El BPC en PC es raramente efectiva para el manejo del dolor a largo plazo.
- Los pseudoquistes deben ser intervenidos solo si se piensa que causan dolor u obstrucción intestinal.

- La compresión del ducto biliar común se trata con stents a largo plazo.

\section{REFERENCIAS}

1. Domínguez Muñoz JE, Lucendo Villarín AJ, Carballo Álvarez LF, Tenías JM, Iglesias García J. Spanish multicenter study to estimate the incidence of chronic pancreatitis. Rev Esp Enferm Dig. 2016;108(7):411-6. doi: 10.17235/ reed.2016.4056/2015.

2. Lizarazo-Rodríguez JI. Fisiopatología de la pancreatitis crónica. Rev Col Gastroenterol. 2008;23:290-8.

3. Majumder S, Chari ST. Chronic pancreatitis. Lancet. 2016;387(10031):1957-66. doi: 10.1016/S01406736(16)00097-0.

4. Yang D, Forsmark CE. Chronic pancreatitis. Curr Opin Gastroenterol. 2017;33(5):396-403. doi: 10.1097/ MOG.0000000000000377.

5. Talukdar R, Reddy DN. Pain in chronic pancreatitis: managing beyond the pancreatic duct. World J Gastroenterol. 2013;19(38):6319-28. doi: 10.3748/wjg.v19.i38.6319.

6. Dumonceau JM, Delhaye M, Tringali A, Dominguez-Munoz JE, Poley JW, Arvanitaki M, et al. Endoscopic treatment of chronic pancreatitis: European Society of Gastrointestinal Endoscopy (ESGE) Clinical Guideline. Endoscopy. 2012;44(8):784-800. doi: 10.1055/s-0032-1309840.

7. Kirkegård J, Mortensen FV, Cronin-Fenton D. Chronic Pancreatitis and Pancreatic Cancer Risk: A Systematic Review and Meta-analysis. Am J Gastroenterol. 2017;112(9):1366-1372. doi: 10.1038/ajg.2017.218.

8. Issa Y, Kempeneers MA, van Santvoort HC, Bollen TL, Bipat S, Boermeester MA. Diagnostic performance of imaging modalities in chronic pancreatitis: a systematic review and meta-analysis. Eur Radiol. 2017;27(9):3820-3844. doi: 10.1007/s00330-016-4720-9.

9. Hall TC, Garcea G, Webb MA, Al-Leswas D, Metcalfe MS, Dennison AR. The socio-economic impact of chronic pancreatitis: a systematic review. J Eval Clin Pract. 2014;20(3):203-7. doi: 10.1111/jep.12117.

10. Korpela T, Udd M, Tenca A, Lindström O, Halttunen J, Myrskysalo S, et al. Long-term results of combined ESWL and ERCP treatment of chronic calcific pancreatitis. Scand J Gastroenterol. 2016;51(7):866-71. doi: 10.3109/00365521.2016.1150502.

11. Talukdar R, Reddy DN. Pancreatic Endotherapy for Chronic Pancreatitis. Gastrointest Endosc Clin N Am. 2015;25(4):765-77. doi: 10.1016/j.giec.2015.06.010.

12. Adler JM, Gardner TB. Endoscopic Therapies for Chronic Pancreatitis. Dig Dis Sci. 2017;62(7):1729-1737. doi: 10.1007/s10620-017-4502-5.

13. Okuno N, Hara K, Mizuno N, Hijioka S, Kuwahara T, Fujita A, et al. Advanced technique for the treatment of chronic calculous pancreatitis using endoscopic ultra- 
sound-guided pancreatic duct drainage. Endoscopy. 2017;49(8):E197-E199. doi: 10.1055/s-0043-110666.

14. Moura RN, De Moura EG, Bernardo WM, Otoch JP, Bustamante FA, Albers DV, et al. Endoscopic-ultrasound versus percutaneous-guided celiac plexus block for chronic pancreatitis pain. A systematic review and meta-analysis. Rev Gastroenterol Peru. 2015;35(4):333-41.

15. Kim YH, Jang SI, Rhee K, Lee DK. Endoscopic treatment of pancreatic calculi. Clin Endosc. 2014;47(3):227-35. doi: 10.5946/ce.2014.47.3.227.

16. Tandan M, Talukdar R, Reddy DN. Management of Pancreatic Calculi: An Update. Gut Liver. 2016;10(6):873880. doi: $10.5009 /$ gnl15555.

17. Ang TL. Chronic pancreatitis with pancreatic duct stricture and calculi treated by fully covered self-expandable metal stent placement and intraductal pancreatoscopy-guided laser lithotripsy. Endoscopy. 2017;49(6):E145-E146. doi: 10.1055/s-0043-105570.

18. Bekkali NL, Murray S, Johnson GJ, Bandula S, Amin $\mathrm{Z}$, Chapman $\mathrm{MH}$, et al. Pancreatoscopy-Directed Electrohydraulic Lithotripsy for Pancreatic Ductal Stones in Painful Chronic Pancreatitis Using SpyGlass. Pancreas. 2017;46(4):528-530. doi: 10.1097/ MPA.0000000000000790.

19. Castaño R, Puerta JD, Ruiz M, Hoyos S. Cirugía de Frey para la pancreatitis crónica. Rev Col Cirugía. 2003;18:13947.

20. TalukdarR,NageshwarReddyD.Endoscopictherapyforchronic pancreatitis. Curr Opin Gastroenterol. 2014;30(5):4849. doi: 10.1097/MOG.0000000000000091.

21. Cahen DL, van der Merwe SW, Laleman W, PoleyJW, Bruno MJ. A biodegradable non-covered self-expandable stent to treat pancreatic duct strictures in chronic pancreatitis: a proof of principle. Gastrointest Endosc. 2018;87(2):486491. doi: 10.1016/j.gie.2017.08.018.

22. Ohyama $H$, Mikata R, Ishihara T, Sakai Y, Sugiyama H, Yasui $S$, et al. Efficacy of multiple biliary stenting for refractory benign biliary strictures due to chronic calcifying pancreatitis. World J Gastrointest Endosc. 2017;9(1):12-18. doi: 10.4253/wjge.v9.i1.12.

23. Tyberg A, Sharaiha RZ, Kedia P, Kumta N, Gaidhane M, Artifon E, et al. EUS-guided pancreatic drainage for pancreatic strictures after failed ERCP: a multicenter international collaborative study. Gastrointest Endosc. 2017;85(1):164169. doi: 10.1016/j.gie.2016.07.030.

24. Hao L, Pan J, Wang D, Bi YW, Ji JT, Xin Let al. Risk factors and nomogram for pancreatic pseudocysts in chronic pancreatitis: A cohort of 1998 patients. J Gastroenterol Hepatol. 2017;32(7):1403-1411. doi: 10.1111/jgh.13748.

25. Pang TC, Maher R, Gananadha S, Hugh TJ, Samra JS. Peripancreatic pseudoaneurysms: a management-based classification system. Surg Endosc. 2014;28(7):2027-38. doi: 10.1007/s00464-014-3434-9.

26. Amin S, Yang DJ, Lucas AL, Gonzalez S, DiMaio CJ. There Is No Advantage to Transpapillary Pancreatic Duct Stenting for the Transmural Endoscopic Drainage of
Pancreatic Fluid Collections: A Meta-Analysis. Clin Endosc. 2017;50(4):388-394. doi: 10.5946/ce.2016.091.

27. Ge PS, Weizmann M, Watson RR. Pancreatic Pseudocysts: Advances in Endoscopic Management. Gastroenterol Clin North Am. 2016;45(1):9-27. doi: 10.1016/j. gtc.2015.10.003.

28. Larsen M, Kozarek RA. Management of Disconnected Pancreatic Duct Syndrome. Curr Treat Options Gastroenterol. 2016;14(3):348-59. doi: 10.1007/s11938016-0098-7.

29. Guo J, Saftoiu A, Vilmann P, Fusaroli P, Giovannini M, Mishra G, et al. A multi-institutional consensus on how to perform endoscopic ultrasound-guided peri-pancreatic fluid collection drainage and endoscopic necrosectomy. Endosc Ultrasound. 2017;6(5):285-291. doi: 10.4103/eus. eus_85_17.

30. Alāi A, Mosko J, May G, Teshima C. Endoscopic Ultrasound-Guided Management of Pancreatic Fluid Collections: Update and Review of the Literature. Clin Endosc. 2017;50(2):117-125. doi: 10.5946/ce.2017.045.

31. Oza VM, Kahaleh M. Endoscopic management of chronic pancreatitis. World J Gastrointest Endosc. 2013;5(1):19-28. doi: 10.4253/wjge.v5.i1.19.

32. Saluja SS, Kalayarasan R, Mishra PK, Srivastava S, Chandrasekar S, Godhi S. Chronic pancreatitis with benign biliary obstruction: management issues. World J Surg. 2014;38(9):2455-9. doi: 10.1007/s00268-014-2581-4.

33. Harada N, Kouzu T, Arima M, Asano T, Kikuchi T, Isono K. Endoscopic ultrasound-guided pancreatography: a case report. Endoscopy. 1995;27(8):612-5. doi: 10.1055/s2007-1005769.

34. Chapman CG, Waxman I, Siddiqui UD. Endoscopic Ultrasound (EUS)-Guided Pancreatic Duct Drainage: The Basics of When and How to Perform EUS-Guided Pancreatic Duct Interventions. Clin Endosc. 2016;49(2):161-7. doi: 10.5946/ce.2016.011.

35. Shimamura Y, Mosko J, Teshima C, May GR. Endoscopic Ultrasound-Guided Pancreatic Duct Intervention. Clin Endosc. 2017;50(2):112-116. doi: 10.5946/ce.2017.046.

36. Sabbagh LC, Aponte D, Cañadas R, Torres M, Álvarez E, Prieto $\mathrm{R}$, et al. Guía de práctica clínica para el uso de ultrasonido endoscópico en pancreatitis crónica, lesiones quísticas y sólidas del páncreas en adultos. Rev Col Gastroenterol 2015;30(Supl 1):97-104.

37. Sey MS, Schmaltz L, Al-Haddad MA, DeWitt JM, Calley CS, Juan $M$, et al. Effectiveness and safety of serial endoscopic ultrasound-guided celiac plexus block for chronic pancreatitis. Endosc Int Open. 2015;3(1):E56-9. doi: 10.1055/s0034-1377919.

38. Gress F, Schmitt C, Sherman S, Ciaccia D, Ikenberry S, Lehman G. Endoscopic ultrasound-guided celiac plexus block for managing abdominal pain associated with chronic pancreatitis: a prospective single center experience. Am J Gastroenterol. 2001;96(2):409-16. doi: 10.1111/j.15720241.2001.03551.x. 
39. Fusaroli P, Caletti G. Is there a role for celiac plexus block for chronic pancreatitis? Endosc Int Open. 2015;3(1):E60-2. doi: 10.1055/s-0034-1391392.

40. Li BR, Liao Z, Du TT, Ye B, Zou WB, Chen H, et al. Risk factors for complications of pancreatic extracorporeal shock wave lithotripsy. Endoscopy. 2014;46(12):1092-100. doi: $10.1055 / \mathrm{s}-0034-1377753$.

41. Samuelson A, Zeligman B, Russ P, Austin GL, Yen R, Shah RJ. Pancreatic Duct Changes in Patients With Chronic Pancreatitis Treated With Polyethylene and Sof-FlexMaterial Stents: A Blinded Comparison. Pancreas. 2016;45(2):2815. doi: 10.1097/MPA.0000000000000471.

42. Zerem E, Hauser G, Loga-Zec S, Kunosić S, Jovanović P, Crnkić D. Minimally invasive treatment of pancreatic pseudocysts. World J Gastroenterol. 2015;21(22):6850-60. doi: 10.3748/wjg.v21.i22.6850.

43. Gimeno-García AZ, Elwassief A, Paquin SC, Sahai AV. Fatal complication after endoscopic ultrasound-guided celiac plexus neurolysis. Endoscopy. 2012;44 Suppl 2 UCTN:E267. doi: 10.1055/s-0032-1309709.

44. Ohyama H, Mikata R, Ishihara T, Tsuyuguchi T, Sakai Y, Sugiyama $\mathrm{H}$, et al. Efficacy of stone density on noncontrast computed tomography in predicting the outcome of extracorporeal shock wave lithotripsy for patients with pancreatic stones. Pancreas. 2015;44(3):422-8. doi: 10.1097/ MPA.0000000000000277.

45. Brand B, Kahl M, Sidhu S, Nam VC, Sriram PV, Jaeckle S, et al. Prospective evaluation of morphology, function, and quality of life after extracorporeal shockwave lithotripsy and endoscopic treatment of chronic calcific pancreatitis. Am J Gastroenterol. 2000;95(12):3428-38. doi: 10.1111/j.15720241.2000.03190.x.

46. Kozarek RA, Brandabur JJ, Ball TJ, Gluck M, Patterson DJ, Attia $\mathrm{F}$, et al. Clinical outcomes in patients who undergo extracorporeal shock wave lithotripsy for chronic calcific pancreatitis. Gastrointest Endosc. 2002;56(4):496-500. doi: $10.1067 /$ mge.2002.128105.

47. Farnbacher MJ, Schoen C, Rabenstein T, Benninger J, Hahn EG, Schneider HT. Pancreatic duct stones in chronic pancreatitis: criteria for treatment intensity and success. Gastrointest Endosc. 2002;56(4):501-6. doi: 10.1067/ mge.2002.128162.

48. Delhaye M, Arvanitakis M, Verset G, Cremer M, Devière J. Long-term clinical outcome after endoscopic pancreatic ductal drainage for patients with painful chronic pancreatitis. Clin Gastroenterol Hepatol. 2004;2(12):1096-106.

49. Inui $K$, Tazuma S, Yamaguchi $T$, Ohara $H$, Tsuji $T$, Miyagawa $\mathrm{H}$, et al. Treatment of pancreatic stones with extracorporeal shock wave lithotripsy: results of a multicenter survey. Pancreas. 2005;30(1):26-30.

50. Dumonceau JM, Costamagna G, Tringali A, Vahedi K, Delhaye M, Hittelet A, et al. Treatment for painful calcified chronic pancreatitis: extracorporeal shock wave lithotripsy versus endoscopic treatment: a randomised controlled trial. Gut. 2007;56(4):545-52. doi: 10.1136/gut.2006.096883.
51. Seven G, Schreiner MA, Ross AS, Lin OS, Gluck M, Gan SI, et al. Long-term outcomes associated with pancreatic extracorporeal shock wave lithotripsy for chronic calcific pancreatitis. Gastrointest Endosc. 2012;75(5):997-1004. e1. doi: 10.1016/j.gie.2012.01.014.

52. Tandan M, Reddy DN, Talukdar R, Vinod K, Santosh D, Lakhtakia $S$, et al. Long-term clinical outcomes of extracorporeal shockwave lithotripsy in painful chronic calcific pancreatitis. Gastrointest Endosc. 2013;78(5):726-33. doi: 10.1016/j.gie.2013.05.012.

53. Vaysse T, Boytchev I, Antoni G, Croix DS, Choury $\mathrm{AD}$, Laurent $\mathrm{V}$, et al. Efficacy and safety of extracorporeal shock wave lithotripsy for chronic pancreatitis. Scand J Gastroenterol. 2016;51(11):1380-5. doi: 10.1080/00365521.2016.1209688.

54. Choi EK, McHenry L, Watkins JL, Sherman S, Fogel EL, Coté GA, et al. Use of intravenous secretin during extracorporeal shock wave lithotripsy to facilitate endoscopic clearance of pancreatic duct stones. Pancreatology. 2012;12(3):272-5. doi: 10.1016/j.pan.2012.02.012.

55. Morgan DE, Smith JK, Hawkins K, Wilcox CM. Endoscopic stent therapy in advanced chronic pancreatitis: relationships between ductal changes, clinical response, and stent patency. Am J Gastroenterol. 2003;98(4):821-6. doi: 10.1111/j.1572-0241.2003.07381.x.

56. Vitale GC, Cothron K, Vitale EA, Rangnekar N, Zavaleta CM, Larson GM, et al. Role of pancreatic duct stenting in the treatment of chronic pancreatitis. Surg Endosc. 2004;18(10):1431-4. doi: 10.1007/s00464-003-8933-z.

57. Eleftherladis N, Dinu F, Delhaye M, Le Moine O, Baize $\mathrm{M}$, Vandermeeren A, et al. Long-term outcome after pancreatic stenting in severe chronic pancreatitis. Endoscopy. 2005;37(3):223-30.

58. Ishihara T, Yamaguchi T, Seza K, Tadenuma H, Saisho H. Efficacy of s-type stents for the treatment of the main pancreatic duct stricture in patients with chronic pancreatitis. Scand J Gastroenterol. 2006;41(6):744-50. doi: $10.1080 / 00365520500383597$.

59. Weber A, Schneider J, Neu B, Meining A, Born P, Schmid RM, et al. Endoscopic stent therapy for patients with chronic pancreatitis: results from a prospective followup study. Pancreas. 2007;34(3):287-94. doi: 10.1097/ mpa.0b013e3180325ba6.

60. Park DH, Kim MH, Moon SH, Lee SS, Seo DW, Lee SK. Feasibility and safety of placement of a newly designed, fully covered self-expandable metal stent for refractory benign pancreatic ductal strictures: a pilot study (with video). Gastrointest Endosc. 2008;68(6):1182-9. doi: 10.1016/j. gie.2008.07.027.

61. Moon SH, Kim MH, Park DH, Song TJ, Eum J, Lee SS, et al. Modified fully covered self-expandable metal stents with antimigration features for benign pancreatic-duct strictures in advanced chronic pancreatitis, with a focus on the safety profile and reducing migration. Gastrointest Endosc. 2010;72(1):86-91. doi: 10.1016/j.gie.2010.01.063. 
62. Seza K, Yamaguchi T, Ishihara T, Tadenema H, Tawada K, Saisho $\mathrm{H}$, et al. A long-term controlled trial of endoscopic pancreatic stenting for treatment of main pancreatic duct stricture in chronic pancreatitis. Hepatogastroenterology. 2011;58(112):2128-31. doi: 10.5754/hge09346.

63. Giacino C, Grandval P, Laugier R. Fully covered self-expanding metal stents for refractory pancreatic duct strictures in chronic pancreatitis. Endoscopy. 2012;44(9):874-7. doi: 10.1055/s-0032-1309774.

64. Weber A, Schneider J, Neu B, Meining A, Born P, von Delius $S$, et al. Endoscopic stent therapy in patients with chronic pancreatitis: a 5-year follow-up study. World J Gastroenterol. 2013;19(5):715-20. doi: 10.3748/wjg.v19.i5.715.

65. Ogura T, Onda S, Takagi W, Kitano M, Sano T, Okuda A, et al. Placement of a $6 \mathrm{~mm}$, fully covered metal stent for main pancreatic head duct stricture due to chronic pancreatitis: a pilot study (with video). Therap Adv Gastroenterol. 2016;9(5):722-8. doi: 10.1177/1756283X16651855.

66. Shen Y, Liu M, Chen M, Li Y, Lu Y, Zou X. Covered metal stent or multiple plastic stents for refractory pancreatic ductal strictures in chronic pancreatitis: a systematic review. Pancreatology. 2014;14(2):87-90. doi: 10.1016/j. pan.2013.12.005.

67. Costamagna G, Bulajic M, Tringali A, Pandolfi M, Gabbrielli A, Spada C, et al. Multiple stenting of refractory pancreatic duct strictures in severe chronic pancreatitis: long-term results. Endoscopy. 2006;38(3):254-9. doi: 10.1055/s2005-921069.

68. Varadarajulu S, Bang JY, Sutton BS, Trevino JM, Christein JD, Wilcox CM. Equal efficacy of endoscopic and surgical cystogastrostomy for pancreatic pseudocyst drainage in a randomized trial. Gastroenterology. 2013;145(3):583-90. e1. doi: 10.1053/j.gastro.2013.05.046.

69. Arvanitakis M, Delhaye M, Bali MA, Matos C, De Maertelaer V, Le Moine O, et al. Pancreatic-fluid collections: a randomized controlled trial regarding stent removal after endoscopic transmural drainage. Gastrointest Endosc. 2007;65(4):60919. doi: 10.1016/j.gie.2006.06.083.

70. Banks PA, Bollen TL, Dervenis C, Gooszen HG, Johnson $\mathrm{CD}$, Sarr MG, et al. Classification of acute pancreatitis--2012: revision of the Atlanta classification and defini- tions by international consensus. Gut. 2013;62(1):102-11. doi: 10.1136/gutjnl-2012-302779.

71. Acevedo-Piedra NG, Moya-Hoyo N, Rey-Riveiro M, Gil S, Sempere L, Martínez J, et al. Validation of the determinantbased classification and revision of the Atlanta classification systems for acute pancreatitis. Clin Gastroenterol Hepatol. 2014;12(2):311-6. doi: 10.1016/j.cgh.2013.07.042.

72. Arslanlar S, Jain R. Benign biliary strictures related to chronic pancreatitis: balloons, stents, or surgery. Curr Treat Options Gastroenterol. 2007;10(5):369-75.

73. van Boeckel PG, Vleggaar FP, Siersema PD. Plastic or metal stents for benign extrahepatic biliary strictures: a systematic review. BMC Gastroenterol. 2009;9:96. doi: 10.1186/1471230X-9-96.

74. Lawrence C, Romagnuolo J, Payne KM, Hawes RH, Cotton PB. Low symptomatic premature stent occlusion of multiple plastic stents for benign biliary strictures: comparing standard and prolonged stent change intervals. Gastrointest Endosc. 2010;72(3):558-63. doi: 10.1016/j. gie.2010.05.029.

75. Khan MA, Baron TH, Kamal F, Ali B, Nollan R, Ismail MK, et al. Efficacy of self-expandable metal stents in management of benign biliary strictures and comparison with multiple plastic stents: a meta-analysis. Endoscopy. 2017;49(7):682694. doi: 10.1055/s-0043-109865.

76. Shah JN, Marson F, Weilert F, Bhat YM, Nguyen-Tang T, et al. Single-operator, single-session EUS-guided anterograde cholangiopancreatography in failed ERCP or inaccessible papilla. Gastrointest Endosc. 2012;75(1):56-64. doi: 10.1016/j.gie.2011.08.032.

77. Vila JJ, Pérez-Miranda M, Vazquez-Sequeiros E, Abadia MA, Pérez-Millán A, González-Huix F, et al. Initial experience with EUS-guided cholangiopancreatography for biliary and pancreatic duct drainage: a Spanish national survey. Gastrointest Endosc. 2012;76(6):1133-41. doi: 10.1016/j. gie.2012.08.001.

78. Ergun M, Aouattah T, Gillain C, Gigot JF, Hubert C, Deprez $\mathrm{PH}$. Endoscopic ultrasound-guided transluminal drainage of pancreatic duct obstruction: long-term outcome. Endoscopy. 2011;43(6):518-25. doi: 10.1055/s-00301256333. 